\title{
MMW-HOTLINE
}

Leser der MMW können sich mit allen Fragen zur Abrechnung und Praxisführung an Helmut Walbert, Facharzt für Allgemeinmedizin, Würzburg, wenden. Sie erreichen inn jeden Donnerstag von 13 bis 15 Uhr unter der kostenlosen Rufnummer (0800) 2379830 oder per E-Mail: w@lbert.info.

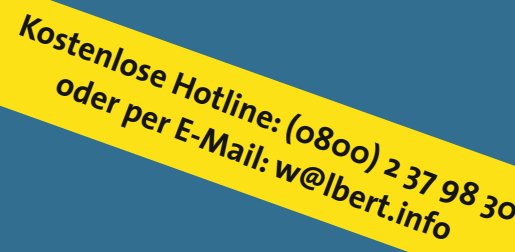

Helmut Walbert

Allgemeinarzt, Medizinjournalist und Betriebswirt Medizin

\section{Warum hat mir die KVB die psychosomatische Abklärung gestrichen?}

Frage von Dr. med. Ch. P., Allgemeinarzt, KV Bayern:

Ich habe regelmäßig vertragsärztlichen Notfalldienst. In diesem Dienst treffe ich häufig auf Patienten mit psychosomatischen Beschwerden. Die Abklärung ist für eine zielgerichtete Intervention sehr wichtig. Ich habe in diesen Fällen die GOP 35100 abgerechnet. Die KVB hat diese Leistung im Rahmen der sachlich und rechnerischen Richtigstellung gestrichen. Ist dies korrekt?

Antwort: Leider ja. Im organisierten Bereitschaftsdienst sind Beratungs-, Gesprächs- und Erörterungsleistungen nicht abrechenbar. Dieser generelle Ausschluss ist sicherlich in den von Ihnen zitierten

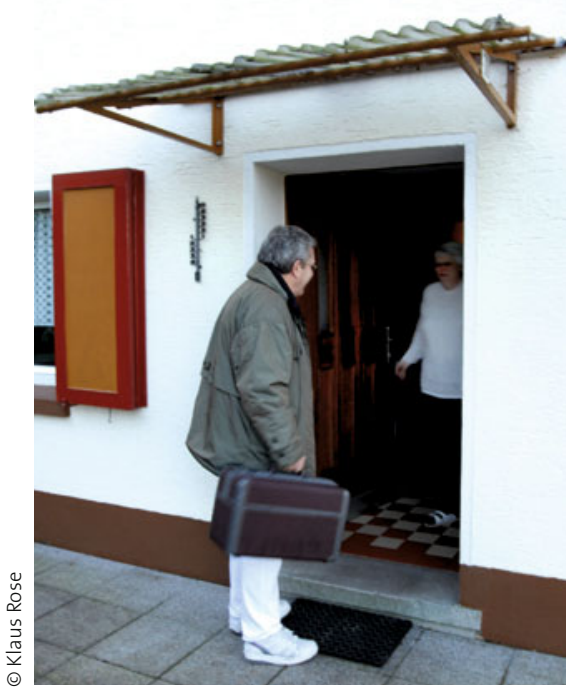

Fällen nicht sachgerecht und vor allem nicht wirtschaftlich im Sinne des $§ 12$ SGB V. Wird der Zusammenhang mit psychischen Ursachen nicht aufgedeckt, kommt es vielfach zu aufwendigen Folgekosten.

Die offizielle Begründung lautet, dass im Rahmen der Notfallversorgung nur solche Leistungen berechnungsfähig sind, die in unmittelbarem diagnostischen oder therapeutischen Zusammenhang mit der Notfall-Versorgung stehen. Dies ist in Ihren Fällen zwar sicherlich gegeben, dennoch kann die Leistung nicht mit der GOP 35100 abgerechnet werden. Der Verordnungsgeber versteht unter „Notfallversorgung" eben nur die unbedingt notwendige Minimalversorgung.

\section{Zuschlag GOP A}

\section{Wann genau ist „außerhalb der Sprechstunde"?}

Frage von Dr.med. F.-J. P., Berlin

Wann ist in der GOÄ eine Behandlung oder Beratung „außerhalb“ der Sprechstunde?

Antwort: Sie haben eine Praxis mit vereinbarten Terminen für die Sprechstunde. Auf dem Praxisschild stehen Zeiten, zu denen Patienten auch ohne Termin die Sprechstunde aufsuchen können. Dann haben wir folgende Situationen: Außerhalb der Zeiten, die als „offen“ auf dem Praxisschild stehen, sind alle Untersuchungen und Beratungen „außerhalb“ der Sprechstunde. In diesen Fällen kann der Zuschlag GOP A

4 Bitte keine Gespräche im Notfalldienst! für Leistungen außerhalb der Sprechstunde zu der jeweiligen Leistung hinzugesetzt werden. Die GOP A darf nur zum einfachen Gebührenordnungssatz und nur einmal je Inanspruchnahme verrechnet werden. Die Berechnung kann auch - im Gegensatz zum EBM - dann erfolgen, wenn die Terminsprechstunde noch läuft oder die normale „offene" Sprechstunde noch abgearbeitet wird.

Die GOP A kann auch bei Besuchen neben den GOP 6 bis 8 angesetzt werden. Allerdings nicht neben der GOP E, dem „Zuschlag für dringend angeforderte und unverzüglich erfolgte Ausführung“. Da die GOP E mit $€$ 9,33 vergütet ist, die GOP A hingegen nur mit $€ 4,08$ ist die Wahl der GOP E ohne Alternative. 